\title{
Optimization of Inter-Cluster Economic and Financial, Information and Logistics Interaction in Conditions of Stochastic Uncertainty
}

\author{
Jashin S.N. \\ Lobachevsky State University of Nizhny Novgorod, \\ Nizhny Novgorod, Russia, \\ jashinsn@yandex.ru
}

\author{
Koshelev E.V. \\ Lobachevsky State University of Nizhny Novgorod, \\ Nizhny Novgorod, Russia, \\ ekoshelev@yandex.ru
}

\begin{abstract}
Simulation technologies based on the principles of stochastic optimization can bring a significant financial effect in planning the investment development of both individual innovation and industrial clusters and federal districts of the country. In such conditions, it becomes important to study the mechanisms of inter-cluster interaction within a single district. Among the priority directions of this cooperation, we include economic and financial, information and logistics. Creating and debugging a digital twin of such interaction would allow us to solve important strategic tasks of the state, without initially resorting to risky management decisions. The model of stochastic optimization of economic, financial, information and logistics inter-cluster interaction within a single federal district assumes as the main most significant characteristic of the success of such interaction the natural increase in the population of the region in which a particular cluster is located. This model involves the implementation of such stages as selecting and correcting the necessary parameters of the digital twin, building and debugging the statistical model of the twin, debugging the stochastic optimization algorithm of the twin. At the last stage, the appropriate settings for the template search method are used. The result of the presented model is that an increase in investment in fixed assets does not always lead to population growth in the regions of the federal district. This should be taken into account when reallocating investment and human resources within a single federal district.
\end{abstract}

Keywords-stochastic optimization, digital twin, inter-cluster interaction

\section{INTRODUCTION}

Modern methods of managing large innovative systems, such as a region or an entire federal district, require adaptation to new technological challenges that are global in nature. The development and implementation of artificial intelligence is one of these tasks on a national scale. Thus, simulation modeling technologies based on the principles of stochastic optimization can bring a significant financial effect in planning the investment development of individual innovation and industrial clusters, as well as federal districts of the country that include similar natural formations in the field of business and public administration.
In such circumstances, it becomes important to study the mechanisms of inter-cluster interaction within a single district [1]. Among the priority areas of this cooperation, we include economic and financial, information and logistics. Creating and debugging a digital twin of such interaction would allow us to solve important strategic tasks of the state, without initially resorting to risky management decisions. On the contrary, the use of such digital twins would make it possible to test government decisions using computer simulations in order to avoid future severe consequences of erroneous directives.

\section{RESEARCH QUESTIONS}

Stochastic optimization is a class of optimization algorithms that use randomness to find the optimal solution. Randomness can manifest itself in different areas. Stochastic optimization algorithms are usually used if the objective function is composite, multi-extremal, with discontinuities, with interference (noise), and so on.

Currently, there are many different approaches to stochastic optimization. For example, O.A. Izmakova [2] described two new algorithms for the self-learning problem that belong to the class of randomized stochastic approximation algorithms. As an example, we consider the problem of Hebb-Hopfield neural network training and propose a method for solving, based on a variational approach

L. Sakalauskas [3] considered the method of nonlinear stochastic optimization using a series of Monte Carlo samples and proposed a procedure for the algorithm halting, which is based on testing the statistical hypothesis that the gradient of the objective function is equal to zero and its confidence limit value.

S.S. Sysoev [4] for a randomized stochastic optimization algorithm loosened the conditions for consistency of its estimates, considered the orders of accuracy for a finite number of observations, and proposed a new scheme for implementing this algorithm on quantum computers.

A.V. Gasnikov, P.E. Dvurechensky, and Y.E. Nesterov [5] attempted to describe the current state of gradient projection 
methods (including direct methods and component-bycomponent descent methods) for solving convex stochastic optimization problems with an inaccurate oracle (non-random inaccuracy) that produces a stochastic sub-gradient.

As the main method of stochastic optimization that will be used in this study, we will consider the pattern (Search method, Direct Search). Direct search (pattern search) is a method for solving optimization problems that does not require any information about the gradient of the objective function. Unlike more traditional optimization methods that use information about the gradient or higher derivatives to find the optimal point, the forward search algorithm searches for a set of points around the current point, looking for a point where the value of the objective function is lower than the value at the current point. Direct search can be used to solve problems for which the objective function is not differentiable or even continuous [6].

In addition, E.D. Dolan, R.M. Lewis, and V. Torczon [7] studied the local convergence properties of template search methods, complementing the previously established global convergence properties for this class of algorithms. They showed that the step length control parameter that appears in the definition of template search algorithms provides a reliable first-order asymptotic measure of stativity. This provides an analytical justification for the traditional stopping criterion for pattern search methods. Using this first-order static index, we review the global convergence properties of the pattern search and analyze the behavior of the pattern search in the vicinity of an isolated local minimizer.

C. Audet and J.E. Dennis [8] offered a new convergence analysis for a class of generalized pattern search (GPS) methods by E.D. Dolan, R.W. Lewis, and V.J. Torkzon for optimization without constraints and with linear constraints. This analysis is necessary to understand the successful behavior of the algorithm according to hypotheses that are supported by many practical tasks. In particular, even if the objective function is discontinuous or extended, the methods find a limit point with some minimizing properties. Simple examples have shown that the strength of the optimality conditions at the limit point depends not only on the algorithm, but also on the directions it uses and on the smoothness of the objective function at the limit point under consideration. The contribution of this research is to provide a simple convergence analysis that gives detailed information about the relation of optimality conditions to objective smoothness properties and to the defining directions for the algorithm.

T.G. Kolda, R.M. Lewis, and V. Torczon [9] conducted a review of direct search methods, which begins with a brief summary of the history of direct search methods and a review of the special properties of problems for which they are well suited. Then the authors move on to a broad class of methods that provide a unifying framework that produces different convergence results. The underlying principles allow generalization to handle related constraints and linear constraints. Extensions of problems with nonlinear constraints are also discussed.

R.W. Lewis, A. Shepherd, and V. Torczon [10] studied the implementation of a search method without derived generating sets for linearly bounded minimization without assuming undecidability of constraints. Convergence guarantees for generating set search methods require that the set of search directions have certain geometric properties that allow it to approximate a possible region near the current iteration. In the hard case, calculating the search directions corresponds to finding the extreme rays of a cone with a deforming vertex at the source, which is a difficult task. The authors investigated how modern methods of computational geometry can solve this problem in connection with the creation of a search set. They also studied a number of other practical issues of implementing the algorithm, such as careful consideration of equality constraints and the desirability of extending the set of search directions beyond the theoretically minimal set. The implementation behavior for several tasks from the CUTEr test suite is illustrated. It was successful in dealing with problems including several hundred variables under linear constraints.

C. Audet, and J.E. Dennis [11] considered the problem of minimizing an asymmetric function under general asymmetrical constraints when there are no derivatives of objective or constraint functions available. They introduced a mesh adaptive direct search (MADS) class of algorithms that extends the GPS class to allow local exploration, called polling, in an asymptotically dense set of directions in the space of optimization variables. This means that under a certain hypothesis, including weak constraint qualification, MADS can treat constraints using an extreme barrier approach, setting the goal to infinity for infinite points and treating the problem as unbounded. The main result of GPS convergence is the determination of limit points $\hat{x}$ where generalized Clarke derivatives are neo-negative in a finite set of directions, called refinement directions. Although in the unbounded case, neonegative combinations of these directions cover the entire space, the fact that there can only be finally many GPS refinement directions limits the strict justification of the barrier approach to finally many linear constraints for GPS. The main result of this work is that the general MADS framework is flexible enough to allow generating an asymptotically dense set of refining directions along which Clarke derivatives are nonnegative. The authors proposed an example of MADS for which the refining directions are dense in a hypertonic cone at $\hat{x}$ with probability 1 whenever the iterations associated with the refining directions converge to one $\hat{x}$. The MADS instance is compared to the GPS versions on some test issues. The limitation of author's results is also illustrated with examples.

M.A. Abramson et al. [12] introduced a new way to select directions for the MADS algorithms class. The advantages of this new instance of OrthoMADS are that the survey directions are chosen deterministically, ensuring that the results of a given run are repeated and that they are orthogonal to each other, which gives convex cones of skipped directions at each of iterations that are reasonably minimal. The convergence results for OrthoMADS follow directly from those already published for MADS, and they hold deterministically, rather than with probability one, as in the case of LtMADS, i.e. the first instance of MADS. The initial numerical results are quite good for smooth and non-smooth, as well as bounded and unbounded problems.

Finally, the work of A. Hendra and S. Adinugroho [13] in the field of energy and automation technologies, whose main topic is optimization "Model Prediction Control" (MPC), is of particular practical interest. MPC uses many parameters and some of them can be configured automatically using numerical optimization. These parameters are of interest to researchers, since precisely configured parameters give a reliable and stable 
MPC. There are many methods and algorithms that can be used for numerical optimization, and the goal of the work is to provide a benchmark for some methods and algorithms in terms of speed and result.

\section{STUDY PURPOSES}

Stochastic optimization of the operation of the designated digital twins would allow us to work out the most optimal solutions in advance for the evolution of large innovative systems, for example, in the Federal districts of the country.

\section{RESEARCH METHODS DESCRIPTION}

We present a model of stochastic optimization of economic, financial, information and logistics inter-cluster interaction within a single federal district in Fig. 1.

$\begin{aligned} & \text { 1) Selecting and adjusting the } \\ & \text { necessary parameters of the } \\ & \text { digital twin (statistical data) }\end{aligned}$
$\begin{aligned} & \text { 2) Building and debugging a } \\ & \text { statistical model of a digital } \\ & \text { twin (regression model) }\end{aligned}$
$\begin{gathered}\text { 3) Debugging the stochastic } \\ \text { optimization algorithm for the } \\ \text { digital twin (pattern search) }\end{gathered}$

Fig. 1. The model of inter-cluster interaction stochastic optimization

As you can see in the Fig. 1, the model is based on the idea of creating and debugging a digital twin of the mechanism of these types of interaction. Then we will explain in more detail how these types of interaction are related to each other in this digital double. To do this, go to the description of the stages of implementing the model.

Stage 1 -Selecting and adjusting the necessary parameters of the digital twin. The effectiveness of inter-cluster interaction best reflects the natural population growth $(y)$ in the analyzed regions where the corresponding innovation and industrial clusters are located. However, population growth should be made dependent on such factors of the digital twin model that would sufficiently reflect the directions of inter-cluster interaction, which include economic and financial information and logistics types of interaction. These factors include per capita monetary income (per month) $\left(x_{1}\right)$, investment in fixed assets $\left(x_{2}\right)$, and exports minus imports $\left(x_{3}\right)$. The redistribution of these indicators value by the state and business within a particular federal district between the regions where the clusters are located clearly reflects their economic and financial interaction. On top of that, the redistribution of investments in fixed assets naturally occurs from the information interaction of clusters. In addition, if the average per capita income of the population in one region is higher than in another, this will lead to the migration of the corresponding labor force and intellectual capital in the person of the necessary specialists between the regions. Therefore, it is also a consequence of information interaction between clusters. Finally, the logistics interaction of clusters also reflects the processes of migration of capital and qualified personnel between the corresponding regions of the federal district, which is also reflected in the indicator of exports minus imports.
As a result, we will analyze the function of 3 variables $y=f\left(x_{1}, x_{2}, x_{3}\right)$ for planning the redistribution of these resources by the state in cooperation with business in order to increase the natural population growth of the federal district.

Stage 2 - Building and debugging a statistical model of a digital twin. There we will use a multiple nonlinear regression model, and one that will be of the highest quality in terms of the necessary statistical parameters, as well as one that will not contradict logic, since any analysis must be guided by common sense [14]

Stage 3 - Debugging the stochastic optimization algorithm for the digital twin. The main idea of this algorithm is to search for the global maximum value of a function of many variables on a given segment in the presence of stochastic uncertainty in the objective function. The target function is the regression that is most justified from the point of view of econometrics and does not contradict the analyst's common sense. However, this may result in other regression dependencies that are no worse than the objective function from the point of view of the quality of the statistical model. They should be considered as noise elements for the target function under study.

After that, the approximate value of this noise is determined, and the objective function is optimized in the global sense, taking into account the noise. To do this, we will use the pattern search algorithm (Pattern Search, Direct Search). However, its standard settings may not allow you to find the global maximum value of the stochastic function, i.e. there is a risk of "getting stuck" in the local maximum value during simulation. To solve this problem, we will use the following additional template search settings.

Global optimization features include three direct search (pattern search) algorithms called the GPS algorithm, the generating set search (GSS) algorithm, and the MADS algorithm. All of them are template search algorithms that calculate a sequence of points approaching the optimal point. At each step, the algorithm searches for a set of points, called a mesh, around the current point-the point calculated at the previous step of the algorithm. The mesh is formed by adding the current point to the scalar set of a set of vectors called an array. If the pattern search algorithm finds a point in the mesh that improves the target function at the current point, the new point becomes the current point in the next step of the algorithm. The GPS algorithm uses fixed direction vectors. The GSS algorithm is identical to the GPS algorithm, except when there are linear constraints, and when the current point is near the boundary of the linear constraint. The MADS algorithm uses a random selection of vectors to determine the mesh.

Let's consider the process of creating and debugging a digital twin of the inter-cluster interaction mechanism on the example of the Volga Federal District (VFD). According to the list approved by the Government of the Russian Federation, there are 25 pilot innovative territorial clusters in Russia [15]. In this case, we will consider only those regions in which clusters from this list are located in the VFD (Table I).

Stage 1 -Selecting and adjusting the necessary parameters of the digital twin. Using the indicators of the "Statistical review" of the Federal State Statistics Service [16], we group the necessary data on natural population growth, average per capita income (per month), and investment in fixed assets, and exports minus imports for 10 years from 2009 to 2018 . At the 
same time, in order to compare the data, we will adjust the indicators measured in rubles or dollars for inflation (Table II). Thus, we get data in 2018 prices in Table III.

TABLE I. INNOVATIVE TERRITORIAL CLUSTERS IN THE REGIONS OF VFD

\begin{tabular}{|c|l|l|}
\hline \multicolumn{1}{|c|}{ Regions } & \multicolumn{1}{c|}{ Innovative clusters } \\
\hline 1 & $\begin{array}{l}\text { Nizhny Novgorod } \\
\text { region }\end{array}$ & $\begin{array}{l}\text { Nizhny Novgorod industrial innovation cluster } \\
\text { in the automotive and petrochemical industry }\end{array}$ \\
\hline 2 & Republic of Mordovia & $\begin{array}{l}\text { Energy-efficient lighting and intelligent } \\
\text { lighting control systems }\end{array}$ \\
\hline 3 & Ulyanovsk region & $\begin{array}{l}\text { Consortium: Scientific-educational-production } \\
\text { cluster "Ulyanovsk - Avia" }\end{array}$ \\
\cline { 3 - 4 } & Dimitrovgrad nuclear innovation cluster \\
\hline 5 & Perm Krai & Innovative territorial aerospace cluster \\
\hline 6 & Udmurt Republic & $\begin{array}{l}\text { Innovative territorial cluster of rocket engine } \\
\text { building "Technopolis "Novy Zvezdny" }\end{array}$ \\
\cline { 2 - 4 } & Photonics \\
\hline 7 & Republic of Tatarstan & $\begin{array}{l}\text { The Kamsky innovative territorial and } \\
\text { production cluster }\end{array}$ \\
\hline 8 & $\begin{array}{l}\text { Republic } \\
\text { Bashkortostan }\end{array}$ & Petrochemical territorial cluster \\
\hline
\end{tabular}

TABLE II. THE ANNUAL INFLATION RATE (\%)

\begin{tabular}{|c|c|c|c|c|c|c|c|c|c|}
\hline & $\mathbf{2 0 1 0}$ & $\mathbf{2 0 1 1}$ & $\mathbf{2 0 1 2}$ & $\mathbf{2 0 1 3}$ & $\mathbf{2 0 1 4}$ & $\mathbf{2 0 1 5}$ & $\mathbf{2 0 1 6}$ & $\mathbf{2 0 1 7}$ & $\mathbf{2 0 1 8}$ \\
\hline RUB & 8.78 & 6.10 & 6.58 & 6.45 & 11.36 & 12.91 & 5.38 & 2.52 & 4.27 \\
\hline$\$$ & 1.50 & 2.96 & 1.74 & 1.50 & 0.76 & 0.73 & 2.07 & 2.11 & 1.91 \\
\hline
\end{tabular}

TABLE III. REGRESSION MODEL BUILDING WITH 2018 PRICES (CASE FOR REPUBLIC OF TATARSTAN)

\begin{tabular}{|c|c|c|c|c|}
\hline Year & $\begin{array}{c}\text { C / per capita } \\
\text { monetary } \\
\text { income of the } \\
\text { population (per } \\
\text { month) (RUB) } \\
\boldsymbol{x}_{\mathbf{1}}\end{array}$ & $\begin{array}{c}\text { Investments } \\
\text { in fixed } \\
\text { assets } \\
\text { (million } \\
\text { RUB) } \\
\boldsymbol{x}_{\mathbf{2}}\end{array}$ & $\begin{array}{c}\text { Export } \\
\text { minus } \\
\text { import } \\
\text { (\$ million) }\end{array}$ & $\begin{array}{c}\text { Natural } \\
\text { population } \\
\text { growth } \\
\text { (persons) }\end{array}$ \\
\hline 2009 & 28782.0 & 497040.8 & 8170.6 & -1415 \\
\hline 2010 & 30792.5 & 521763.6 & 11221.9 & -762 \\
\hline 2011 & 31867.1 & 620525 & 14488.8 & 3752 \\
\hline 2012 & 35592.0 & 700725.8 & 15484.2 & 9023 \\
\hline 2013 & 36843.6 & 736854.4 & 13959.5 & 10218 \\
\hline 2014 & 37914.6 & 690372.2 & 11861.9 & 9732 \\
\hline 2015 & 35513.6 & 695245.1 & 7452.5 & 10416 \\
\hline 2016 & 35076.3 & 686810.8 & 6861.0 & 10643 \\
\hline 2017 & 33673.0 & 664837.7 & 6496.5 & 4240 \\
\hline 2018 & 33130.0 & 629731.0 & 8223.7 & 1600 \\
\hline
\end{tabular}

Stage 2 - Building and debugging a statistical model of a digital twin. Using data for 8 regions of the VFD, similar to those presented in Table III, the Statistica program obtained two of the most reliable models of multiple nonlinear regression function:

regression function 1 :

$$
\begin{gathered}
y=-290.198+0.036 x_{2}+57.229 \sqrt{x_{1}}-33.052 \sqrt{x_{2}}, \\
R^{2}=0.6196 ;
\end{gathered}
$$

regression function 2 :

$$
\begin{gathered}
y=15.283-148.867 .881 \frac{1}{x_{1}}-35 \sqrt{x_{2}}, \\
R^{2}=0.6201 .
\end{gathered}
$$

However, the Statistica program discarded the $x_{3}$ variable as insignificant.

Let's plot both regressions in the Matlab program on the same graph (Fig. 2). The situation for regression 2 in the lower graph is unnatural, where at the maximum personal income of the maximum population growth will be at zero investment in fixed assets. As you know, without investment in fixed assets, it is impossible to develop innovative and industrial clusters, which, in turn, will lead to a drop in production and a reduction in the income of the population in such a cluster. Then we take regression 1 as the objective function, and regression 2 , the equation of which is shown in Fig. 2, will be further considered as noise.

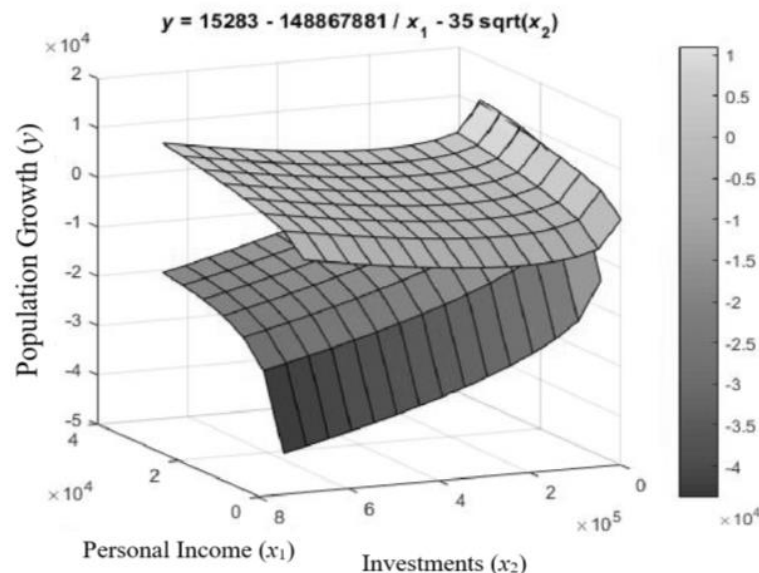

Fig. 2. Graphs of two regressions for 8 regions of the VFD with clusters

Stage 3 - Debugging the stochastic optimization algorithm for the digital twin. To better estimation of the approximate noise range (Fig. 3), we subtract the maximum $y$ value of the noise function, i.e. regression 2 , from the maximum $y$ value of the target function, i.e. regression 1 . They can be found analytically using data marked in italics in Table III.

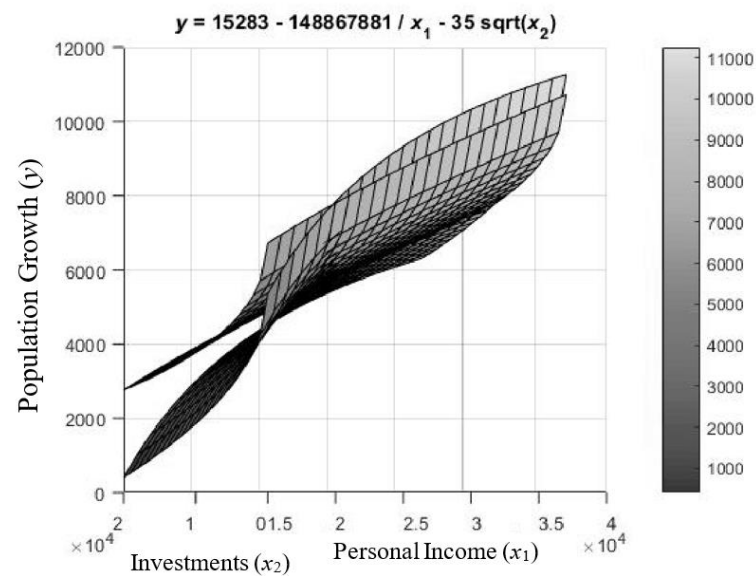

Fig. 3. Maximum values of two regression graphs for 8 regions of the VFD with clusters

So, for regression 1 , we get that investment $x_{2}=0$ and personal income $x_{1, \max }=37914.6$ rubles, then $y_{\max }=$ 
10853.2 ppl. Similarly, for regression 2 , investment $x_{2}=0$ and personal income $x_{1, \max }=37914.6$ rubles, then $y_{\max }=$ $11356.6 \mathrm{ppl}$. Then the noise makes up the difference $\Delta y=$ $11356.6-10853.2=503.4(\mathrm{ppl})$.

Thus, we have the problem of finding the maximum value of the stochastic function $\mathrm{y}$ on the given segments of its arguments: $x_{1} \in[0 ; 37914.6]$ and $x_{2} \in[0 ; 736854.4]$.

The maximum value of a smooth objective function, i.e. regression 1 without noise, can be found in the Matlab program using the direct internal point method [17]. The result for the desired maximum population growth in one region $(y)$ is 10853.2 people, which is demonstrated in Fig. 4. Since Matlab solves only minimization problems, the graph of the objective function is inverted and, accordingly, the objective function is taken with the opposite sign. The solution obtained by the direct internal point method is marked in Fig. 4 as "fmincon solution".

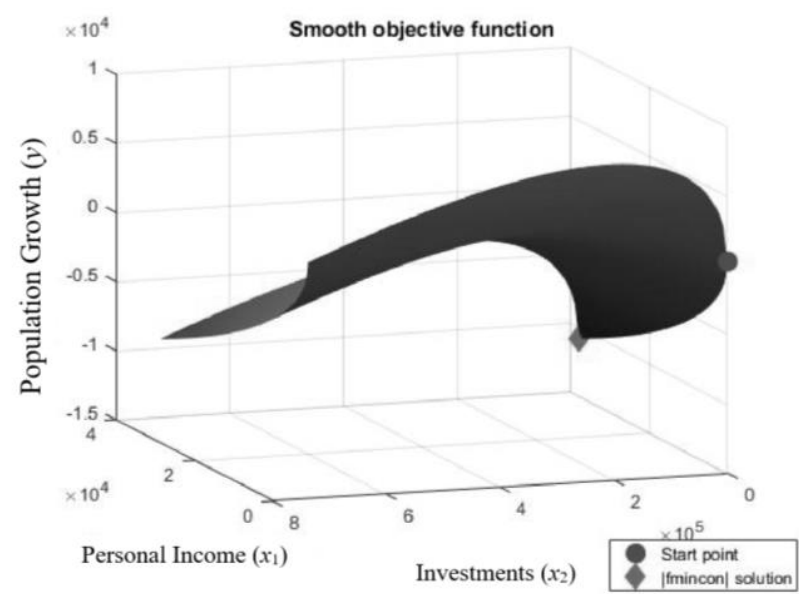

Fig. 4. Search for the minimum value of a smooth objective function

The maximum value of the stochastic objective function, i.e. regression 1 with noise, can be found in the Matlab program using both the direct internal point method (fmincon solution) and the Pattern Search solution (Fig. 5). Thus, the direct internal point method gives the result of optimizing, where $y=31.7$ people and the template search results in $y=381.8$ people, i.e. the local, not global, maximum values of the function are actually found.

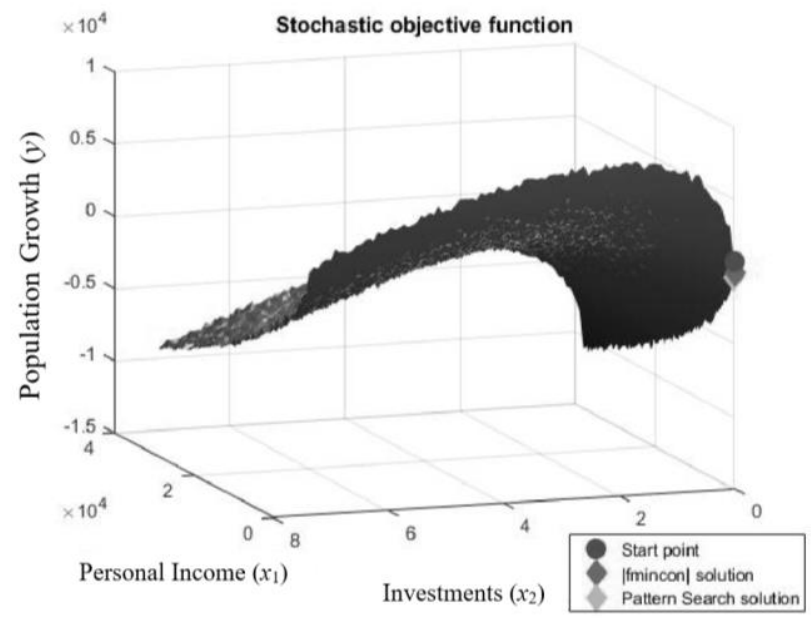

Fig. 5. Search for the minimum value of a stochastic objective function
This problem can be solved by configuring the pattern search algorithm, i.e. using GPS, GSS, and cellular adaptive search (MADS) as the search method and survey method (Table IV). However, it is important that different settings are used for the search and survey methods. In this case, the template search algorithm itself works better. In addition, for all three settings of GPS, GSS and MADS, we will use the $2 N$ configuration, and not, because in the case of $2 N, 6$ closest points are moved relative to the previous one, and only 4 points in the case of $N+1$.

TABLE IV. The Best PATtern SEARCH RESUlts

\begin{tabular}{|c|c|c|c|c|c|c|c|}
\hline 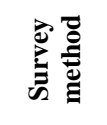 & 丞离 & 总 & 总 & 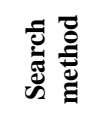 & 駦异 & $F$ & 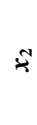 \\
\hline 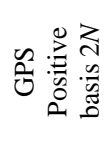 & ธี & 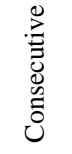 & ธี & 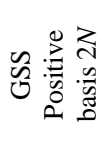 & $\begin{array}{l}\tilde{U} \\
\stackrel{\vec{v}}{=}\end{array}$ & $\underset{\substack{\infty \\
\infty}}{\stackrel{\infty}{n}}$ & $N$ \\
\hline 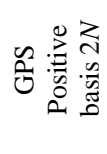 & ธี & 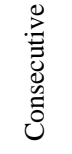 & ฮี & 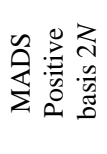 & $\stackrel{m}{=}$ & 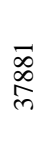 & - \\
\hline 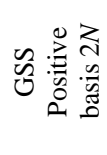 & ธี & 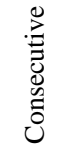 & ฮี & 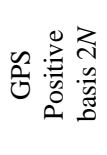 & $\begin{array}{l}\mathfrak{2} \\
\stackrel{2}{=}\end{array}$ & 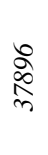 & $?$ \\
\hline 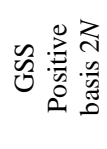 & ธี & $\begin{array}{l}\text { Ḋ } \\
\vdots \\
0 \\
0 \\
0 \\
0\end{array}$ & ธี & 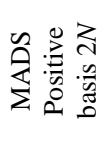 & $\stackrel{\bar{m}}{\underline{\underline{n}}}$ & 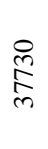 & $\ddot{\circ}$ \\
\hline 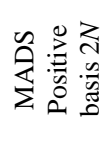 & ธี & 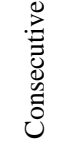 & ธี & 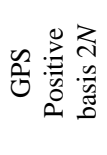 & 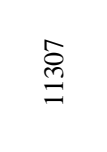 & $\underset{\substack{\infty \\
\infty}}{\stackrel{\infty}{\infty}}$ & 0 \\
\hline 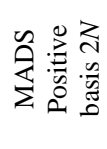 & ธี & 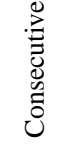 & ฮี & 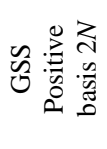 & $\overrightarrow{\bar{m}}$ & $\begin{array}{l}\infty \\
\infty \\
\infty \\
\stackrel{m}{m}\end{array}$ & $n$ \\
\hline
\end{tabular}

As a result, Table IV shows that the maximum value of the stochastic target function of population growth in one region $(y)$ is observed for the "GPS Positive basis $2 N$ " search method and the "GSS Positive basis $2 N$ " subsequent survey method. In this case, the maximum achievable population growth will be 11365 people. This is somewhat more than what was obtained for functions with noise, i.e., regression 2. There were 11356.6 people. This happened because the noise itself is noisy. However, this maximum population growth in the region is possible with investments in fixed assets $x_{2}=0.5$ million rubles; and income of the population per month $x_{1}=37896$ rubles (Table IV).

An important result of the considered model of stochastic optimization of economic, financial, information and logistics inter-cluster interaction is that an increase in investment in fixed assets does not always lead to population growth in the regions of the federal district (Fig. 2, Fig. 5). The population of 
innovative and industrial clusters is primarily interested in the growth of their average per capita income. This should be taken into account when reallocating investment and human resources within a single federal district. A temporary decline in investment in fixed capital and the simultaneous increase in incomes in a particular region of one federal district can lead to more serious growth of population in the region compared to a situation where the lack of income of the population is made up by the growth of investment in fixed capital.

\section{CONCLUSION}

We will formulate the most significant conclusions obtained as a result of the study.

1) Stochastic optimization of the operation of digital twins of inter-cluster interaction allows working out optimal solutions in advance regarding the evolution of large innovative systems, for example, in the federal districts of the country.

2) The model of stochastic optimization of economic, financial, information and logistics inter-cluster interaction within a single federal district assumes as the most significant characteristic of the success of such interaction the natural increase in the population of the region in which a particular cluster is located

3) Using the digital twin of the inter-cluster interaction mechanism will allow avoiding premature unjustified management decisions of the state scale regarding the further development of innovation and industrial clusters located on the territory of a specific federal district of Russia, and, on the contrary, simulate the mechanism of inter-cluster interaction on a computer in the form of a corresponding digital twin. This will then allow debugging the specified digital twin so as to obtain the optimal management decision in terms of global stochastic optimization, and then take it in practice with the possibility of planning and adjusting future government decisions on a computer, using the already debugged digital twin.

\section{Acknowledgement}

The research was carried out with the financial support of the RFBR in the framework of the research project No 19-01000932 "Creating the innovation system evolution model of industrial regions in modern conditions of socio-economic development".

\section{References}

[1] S.N. Yashin, E.V. Koshelev, and R.V. Kostrigin, "Compilation of linear functional of the value of the innovation and industrial cluster for the region", Management of Economic Systems, 2019, vol. 12(130), p. 90. (In Russ.).

[2] O.A. Izmakova, "Randomized self-learning algorithms for setting up associative neural networks" ["Randomizirovannye algoritmy samoobucheniya dlya nastrojki associativnyh nejronnyh setej”], Stochastic Optimization in Computer Science [Stohasticheskaya optimizaciya v informatike], 2005, vol. 1, pp. 81-102. (In Russ.).

[3] L. Sakalauskas, "Nonlinear stochastic optimization by the Monte Carlo method" ["Nelinejnaya stohasticheskaya optimizaciya metodom MonteKarlo"], Stochastic Optimization in Computer Science [Stohasticheskaya optimizaciya v informatike], 2005, vol. 1, pp. 190-205. (In Russ.).

[4] S.S. Sysoev, "Randomized stochastic optimization algorithms, quantum computers, artificial intelligence" ["Randomizirovannye algoritmy stohasticheskoj optimizacii, kvantovye komp'yutery, iskusstvennyj intellekt"], Stochastic Optimization in Computer Science [Stohasticheskaya optimizaciya v informatike], 2005, vol. 1, pp. 206-221. (In Russ.).

[5] A.V. Gasnikov, P.E. Dvurechensky, and Y.E. Nesterov, "Stochastic gradient methods with inexact oracle", Proceedings of MIPT, 2016, vol. 8(1), pp. 41-91. (In Russ.).

[6] T.G. Kolda, R.M. Lewis, and V. Torczon, A generating set direct search augmented Lagrangian algorithm for optimization with a combination of general and linear constraints, Sandia National Laboratories, 2006, 44 p.

[7] E.D. Dolan, R.M. Lewis, and V. Torczon, "On the local convergence of pattern search", SIAM Journal on Optimization, 2003, vol. 14(2), pp. $567-$ 583. DOI: https://doi.org/10.1137/S1052623400374495

[8] C. Audet, and J.E. Dennis, "Analysis of generalized pattern searches", SIAM Journal on Optimization, 2000, vol. 13(3), pp. 889-903. DOI: https://doi.org/10.1137/S1052623400378742

[9] T.G. Kolda, R.M. Lewis, and V. Torczon, "Optimization by direct search: New perspectives on some classical and modern methods", SIAM Review, 2003, vol. 45(3), pp. 385-482. DOI: https://doi.org/10.1137/S0036144502428893

[10] R.M. Lewis, A. Shepherd, and V. Torczon, "Implementing generating set search methods for linearly constrained minimization", SIAM Journal on Scientific Computing, 2007, vol. 29(6), pp. 2507-2530. DOI: https://doi.org/10.1137/050635432

[11] C. Audet, and J.E. Dennis, "Mesh adaptive direct search algorithms for constrained optimization", SIAM Journal on Optimization, 2006, vol. 17(1), pp. 188-217. DOI: https://doi.org/10.1137/060671267

[12] M.A. Abramson, C. Audet, J.E. Dennis, and S.L. Digabel, "OrthoMADS: A deterministic MADS instance with orthogonal directions", SIAM Journal on Optimization, 2009, vol. 20(2), pp. 948-966. DOI: https://doi.org/10.1137/080716980

[13] A. Hendra, and S. Adinugroho, Matlab Solvers Benchmark for ABB's Model Predictive Control Optimization. Speed vs result, Project in computational science: Report, Uppsala: Uppsala Universitet, 2015, 49 p.

[14] A. Damodaran, Investment Valuation: Tools and Techniques for Determining the Value of Any Asset, New York: John Wiley \& Sons, Inc., 2017, 992 p.

[15] Innovative clusters, HSE Univesity, 2012. (In Russ.). Retrieved fom https://cluster.hse.ru/innovative_clusters

[16] Federal State Statistics Service of the Russian Federation, 2020. (In Russ.). Retrieved fom https://rosstat.gov.ru/

[17] M.S. Babynin, and V.G. Zhadan, "A Primal interior point method for the linear semidefinite programming problem", Computational Mathematics and Mathematical Physics, 2008, vol, 48(10), pp. 1746-176 